\title{
A Comparison of Hyperspectral Image Compression Methods
}

\author{
Astha Puri ${ }^{1}{ }^{*}$, Ershad Sharifahmadian ${ }^{1}$, Shahram Latifi ${ }^{1}$ \\ 1 Department of Electrical \& Computer Engineering, University of Nevada, Las Vegas, Nevada, USA. \\ * Corresponding author. Email: puri@unlv.nevada.edu \\ Manuscript submitted June 30, 2014; accepted November 3, 2014. \\ doi: 10. 17706/ijcee.2014.v6.867
}

\begin{abstract}
The hyperspectral imaging plays an important role in remote sensing. Hyperspectral images include both spectral and spatial redundancies whose exploitation is crucial for compression. Most popular image coding algorithms attempt to transform the image data so that the transformed coefficients are largely uncorrelated. In hyperspectral image compression, wavelets have shown a good adaptability to a wide range of data. Some wavelet-based compression methods have been successfully used for hyper spectral image data. In many applications, karhunen-loève transform (KLT) is the popular approach to decorrelate spectral redundancies. In this paper, a review of efficient compression techniques is done, with more emphasis on binary embedded zerotree wavelet (BEZW), 3D set partitioning embedded block (SPECK) and 3D set partitioning in hierarchical trees (SPIHT). In comparison with the techniques discussed, the BEZW technique has lower computational cost, better performance, high efficiency and simplified coding algorithm.
\end{abstract}

Key words: BEZW, Remote sensing, SPECK, SPIHT, transform coding, wavelet transform.

\section{Introduction}

Compression of hyperspectral images has recently become a popular research area. Hyper spectral imagery or spectral imagery involves observing the same scene at different wavelengths. Typically, each image pixel is represented by hundreds of values, corresponding to various wavelengths. These values correspond to a sampling of the continuous spectrum emitted by the pixel. This sampling of the spectrum at very high resolution allows pixel identification (materials, minerals, gases etc.). The availability of the spectral information for each pixel leads to new applications in all fields that use remote sensing data (agriculture, environment, or military), and can help to improve the understanding of the solar system. Hyper spectral data are in a way similar to video data, where wavelength corresponds to time, but their statistical properties are different: there is no motion between hyper spectral planes but changes in color. Ideally compression should be lossless to ensure preservation of the scientific value of data. However, lossless compression techniques provide compression ratios of about two or three, a limitation which is enforced in the hyper spectral data due to the noise inherently present in high-resolution sensors that generate such data [1]. Near lossless compression becomes an increasingly acceptable choice during the sensor selection, in which the maximum error between the original and decoded image is bounded to a user defined value. The Airborne Visible/Infrared Imaging Spectrometer (AVIRIS) was developed by the NASA Jet Propulsion Laboratory in 1987 and provides spectral images with 224 contiguous bands covering the spectral ranges from 400 to $2500 \mathrm{~nm}$. The unique high spectral resolution has been used in a broad range of scientific research such as 
terrain classification, agricultural monitoring, and military surveillance. The AVIRIS instrument yields files of several gigabytes of data, which are recoded and stored onboard. Therefore, compression of the hyper spectral image is necessary to facilitate storage and transmission [2].

A lot of work has been done in the field of lossless compression of hyperspectral data, where predictionbased [3] and wavelet based techniques have been typically employed. Lossy compression has also been considered wherein the 3D transform coding approach has largely been prevalent [4]. A transform such as the wavelet or KLT can be employed in the spectral dimension, followed by spatial decorrelation. For example, the use of the multicomponent transformation feature of JPEG 2000 part 2 has been very popular [5], [6], as well as approaches based on set partitioning. However, while these approaches achieve extremely good performance, especially at low bit-rates, their computational complexity is too high for onboard compression.

There are various transform based compression techniques, which map signal samples from the spatial/spectral domain into another space to produce useful statistical properties. Some examples are- KLT, Discrete Fourier transform (DFT), Discrete Cosine transform (DCT) and Discrete Wavelet Transform (DWT). Penna et al. [5] compressed hyperspectral images using JPEG 2000 and investigated the performance under different transform techniques including WT, DCT, KLT and various combinations.

In this paper, we study few compression techniques for lossless compression of hyperspectral images. First, we study the BEZW technique, which uses an asymmetric dual-tree BEZW algorithm based on KLT and DWT. Discrete wavelet transform has been widely used in image compression because of its excellent decorrelation ability. Wavelet based compression achieves rate scalability, high compression and progressive transmission [7]. These excellent properties have been possible since the introduction of Shapiro's embedded zero tree wavelet (EZW) coder [8]. The EZW coder is based on significant tree quantization that exploits the similarities between the sub band of the wavelet transform domain and the energy distribution of images through the sub band. Later, Said and Pearlman proposed an improved version known as Set Partitioning in Hierarchical Trees (SPIHT) [9], [10]. SPIHT is one of the most efficient wavelet-based compression algorithms and has become the benchmark for the current coders. Another wavelet-based compression, Set partitioning embedded block coding (SPECK) was introduced later to provide a less complex alternative for an embedded coder. A SPECK coder has a comparable performance to SPIHT but is faster. The partitioning rule in SPECK is block partitioning [11]. Hyper spectral/multispectral images require a 3D coder that will efficiently exploit the inter-band correlation between each frame; hence, some of the popular existing 2D wavelet coders have been extended to 3D to suit these 3D image sources. Thus, 3D SPIHT and 3D SPECK came into the picture. 3D SPECK is considered to be less complex and faster.

The Airborne Visible/Infrared Imaging Spectrometer (AVIRIS) was developed by the NASA Jet Propulsion Laboratory in 1987 and provides spectral images with 224 contiguous bands. In our study, we used the standard AVIRIS images-Lunar Lake, Jasper, Cuprite and Low altitude to analyze various methods. The image size used was $225 \times 225 \times 224$.

The rest of the paper is organized as follows. Section 2 describes the analysis of hyperspectral image compression methods. Discussion is presented in Section 3, Section 4 concludes the paper.

\section{Hyperspectral Image Compression Methods}

\subsection{Dual Tree BEZW Compression}

This technique uses asymmetric dual-tree BEZW algorithm for compression of hyperspectral images. The algorithm has been proposed in [2] and adopts KLT and DWT to achieve 3-D integer reversible hybrid transform and decorrelate spectral and spatial data. The 3-D BEZW algorithm compresses hyperspectral images by implementing progressive bitplane coding.

The KLT is a data-dependent and linear orthogonal transform whose matrix consists of the eigenvectors 
derived from the covariance matrix of the data. It is applied to the spectral dimension of hyper spectral images, removing the redundancy contained within the spectral data. The integer KLT is the integer approximation of KLT, which enables lossless hyperspectral image compression [12]. The IKLT can be implemented on factorized matrices which must be recorded as overhead information for the reverse transform. The authors in [2] utilized the quasi-complete pivoting suggested by Galli and Salzo [13] in the process of matrix factorization.

The 2D-IDWT is applied to each IKLT transformed band to gain energy compaction and self-similarity in the two spatial dimensions. The integer transform is implemented using a lifting scheme. The number of bands with significant energy compaction is typically the top 30-50 bands; thus in [2] they chose to perform the 2D-IDWT on only top 40 bands for all the considered images. The presence of energy compaction in the individual bands will determine the efficiency of transform-based compression. The transform based algorithm scans the transformed image multiple times and encodes the significant coefficients with respect to multiple thresholds. The algorithm is efficient because it is based on the non-overlapping asymmetric tree structures, which interpolate the relations among wavelet coefficients across different scales of sub bands. It is seen in [14] that the longer asymmetrical tree along the spectral dimension results in a larger cluster of coefficients and provides higher compression for hyper spectral images.

After the two integer transforms, the signs and magnitudes of coefficients are encoded separately, as shown in Fig. 1. The authors [13] showed that when performing lossless compression, arithmetic coding results in better performance for the coding of the sign bits. On the other hand, conventional EZW results in more compact results for lossy compression of signs. For the proposed algorithm in [2], the magnitudes of coefficients are encoded through the proposed BEZW algorithm based on the two tree structures.

The BEZW algorithm belongs to progressive bitplane coding that encodes all currently significant bits at the same bitplane together and continues encoding others for the next significant bitplane until reaching the least significant bitplane [15]. In the 3-D-BEZW algorithm, the dominant pass achieves both the significance test and quantization. The dominant pass scans all magnitudes of coefficients in raster order to find the significant coefficients with respect to thresholds and computes residual values and binary sequences.

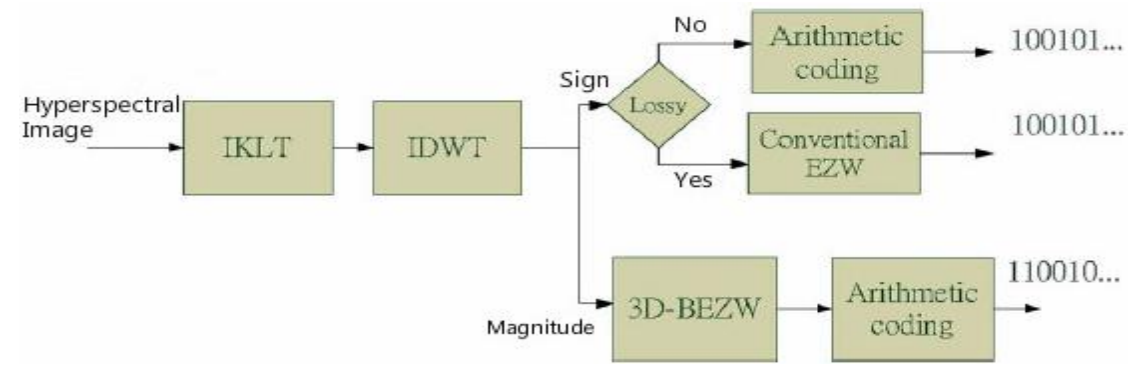

Fig. 1. System diagram for BEZW compression [2].

The initial threshold $T_{0}$ is the highest power of two smaller than the maximum values of coefficients. If a coefficient is recognized as a significant pixel, the coefficient is replaced by a residual value in the image:

$$
R(x, y, z)=I(x, y, z)-T_{i}
$$

where $R(x, y, z)$ is the residual value at $(x, y, z), T_{i}$ is the threshold at the ith iteration, and $I(x, y, z)$ is the magnitude of a pixel at $(x, y, z)[2]$.

In [2] it is stated that each coefficient has two binary outputs. The first binary symbol represents whether the magnitude of a coefficient is larger than the current threshold, and the second represents whether descendant coefficients are significant. The threshold values are set at different values for lossy and lossless 
compression i.e. for lossless BEZW compression the threshold value is 2, and for lossy BEZW compression a predetermined threshold is set. Further efficiency in compression is achieved through subsequent arithmetic coding of the binary sequences as the final step.

\subsection{Compression Using 3D SPECK}

The three dimensional Set Partitioning Embedded bloCK (3D SPECK) algorithm is described in [7] is employed for lossless compression of hyperspectral image. A three dimensional discrete wavelet transform (3D DWT) can fully exploit the inter band correlation in a volumetric block. The 3D structure of the SPECK algorithm extends the exploitation of inters band dependence and correlation. 3D SPECK maintains a block splitting algorithm to sort the significant pixels i.e. if a code block contains significant coefficients it splits into smaller sub-blocks. Also, as hyperspectral images have energy concentration in a high frequency band, 3D SPECK is expected to work well with these images.

3D SPECK maintains two linked lists, List of Insignificant Sets (LIS) and List of Significant Pixels (LSP). The process consists of 4 steps- Initialization, Sorting Pass, Refinement Pass and Quantization step. In the sorting pass, a block splitting method is adopted after a significant test. Refinement pass is the part where some of the coefficient transmitted and the quantization process is continued for the next decreased bit plane until a certain bit rate is achieved. The 3D DWT transformed coefficient de-correlates the hyper spectral image components spatially and spectrally. This brings out some redundancy that can be exploited during the coding process. The proposed coder in [7] follows the basic sorting algorithm as in 3D SPECK. Therefore, inter-band dependence can be exploited automatically. To map the 3D coefficients to a 1D array, the mapping is based on a recursive $Z$ curve or Morton ordering as described in [16] because this performs better at preserving the locality.

Following the linear indexing system, the number of coefficients is stored in a single array of length $I$, where $I=$ Row $\times$ Column $\times$ Frame and is the magnitude array. The state table array, which is based on linear indexing, also has length $I$, with 4 bits per coefficient, which is the marked part. There is a one-to-one correspondence between magnitude and mark. In this proposed algorithm, the memory needed for it is fixed [5].

$S$ is significant with respect to $n$, if

$$
\max _{(i, j, k) \in \mathrm{S}}\left|C_{(i, j, k)}\right| \geq 2^{n}
$$

where $i, j, k, c$ denotes the transformed coefficients at coordinates $(i, j, k)$ otherwise it is significant. The significance can be defined as the function below:

$$
S_{n}(T)=\left\{\begin{array}{l}
1: \text { if } 2^{n} \leq \max _{(i, j, k) \in T}\left|C_{(i, j, k)}\right|<2^{n+1} \\
0: \text { else }
\end{array}\right.
$$

The Hyperspectral images which were tested with the proposed algorithm were obtained from the AVIRIS sensors [17]. The simulation was carried out using MATLAB. The 3D SPECK performs very well on images with high frequency content.

\subsection{Asymmetric Tree (AT) 3D SPIHT Compression}

The SPIHT algorithm was first described by Said and Pearlman in [9]. The algorithm as described in [1] maintains three lists of coefficients: List of Significant Pixels (LSP), List of Insignificant Pixels (LIP) and the List of Insignificant Sets (LIS). In this method, every output is binary and the coefficient is more dependent on the data. The list system of SPIHT leaves the order entirely dependent on the data. Coefficients are processed according to their order in the list. The three-dimensional Set Partitioning in Hierarchical Trees 
(3D SPIHT) based on zero tree coding is a very good candidate for hyper spectral image compression. Also, because the statistics of hyper spectral images are not symmetric along 3 different directions namely spatialhorizontal, spatial-slice and spectral-spatial directions, the performance of the symmetric 3D zero tree structure is not optimal. The authors in [18] applied an asymmetric tree 3D SPIHT algorithm for hyper spectral image and the basic structure of coding system is shown in Fig. 2.

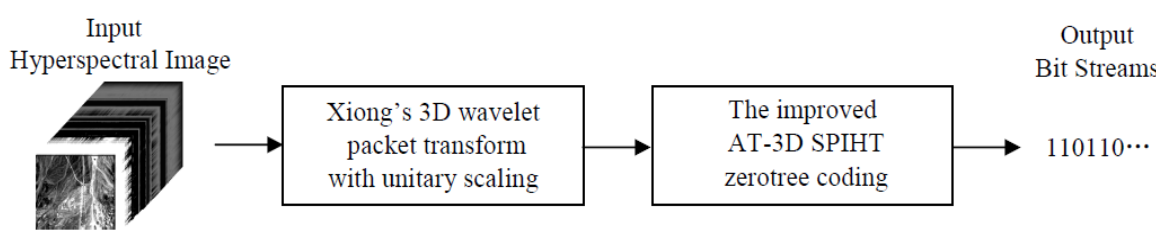

Fig. 2. Block diagram of the AT-3D SPIHT coding system for hyperspectral image compression [18].

The AT 3D SPIHT algorithm for hyper spectral image is proposed in [18]. In this algorithm, the authors constructed a more effective asymmetric 3D zero tree structure which has longer zero tree wavelet. For this algorithm, a more effective asymmetric 3D zero tree structure based on Xiong's 3D integer wavelet packet transform is constructed, which has longer wavelet zero tree and more effective cluster wavelet zero coefficients. Xiong's 3D integer wavelet packet transform (WPT) structure [19] is capable of efficiently utilizing the statistical properties to decorrelate and gaining excellent lossless coding performance for hyperspectral image. It first carries out 1D WPT in the spectral-slice direction, which needs to further decompose the high-frequency component at even decomposition level, and then applies 2D dyadic wavelet transform on each of the resulting spatial image. A unitary scaling structure was adopted.

There are several versions of the 3D SPIHT algorithm based on the diversified 3D wavelet transform structures and the different zero tree structures. AT 3D SPIHT adopted 3D wavelet packet transform and an optimal asymmetric 3D zero tree structure. For the improved AT-3D SPIHT algorithm, a hierarchical pyramidal structure was generated by Xiong's 3D integer WPT with Fig. 3's unitary scaling structure at first. All coefficients are linked to the root coefficients in the lowest spatial and spectral subbands. The zerotree link in the spectral direction is only kept for the lowest spatial subbands, and for each spectral band the zerotree link [20] in the spatial direction is the same as the original 2D SPIHT [21].

The improved AT-3D SPIHT algorithm has the same long wavelet zerotree as the original AT-3D SPIHT algorithm. For the improved AT-3D SPIHT the authors used the embedded zerotree partitioned bitplane coding technique to progressively encode the wavelet coefficients of each subband from the root coefficients to their descendants, until the significant coefficients are located and coded.
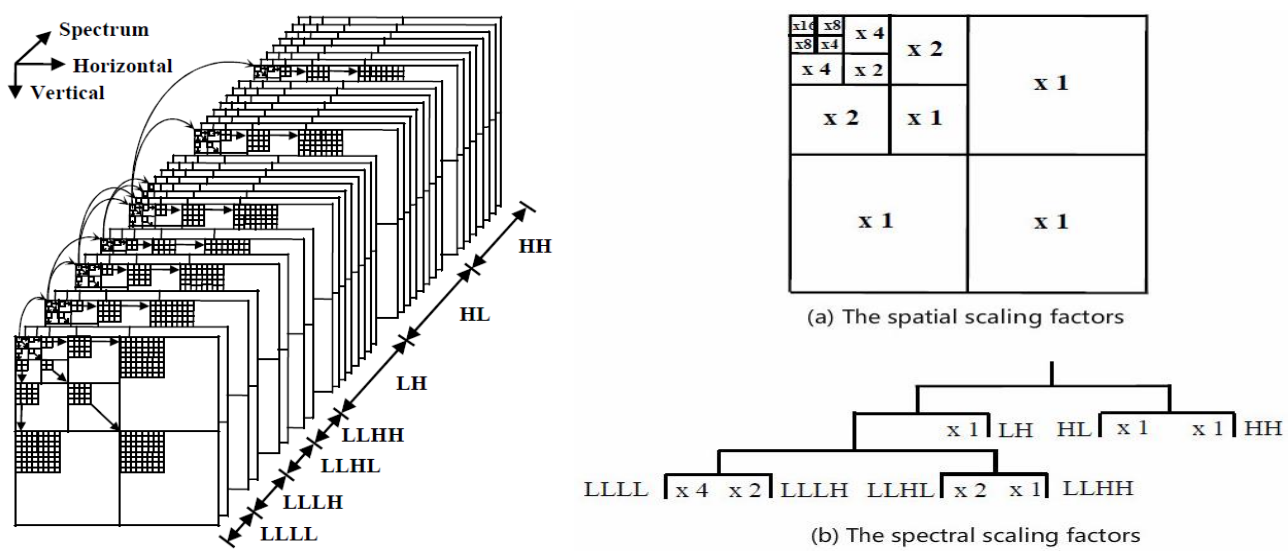

Fig. 3. Xiong's 3D integer WPT structure of four spatial levels and four spectral levels and in (a) and (b) the unitary scaling factors for each subband after the four spatial levels and four spectral levels Xiong's 3D integer WPT [16]. 


\section{Discussion}

There are various categories and criteria to calculate the efficiency of the hyperspectral image compression method. However, a high Signal-to-Noise Ratio (SNR) and a low value of bits per pixel per band (bpppb) are expected. The bpppb for an uncompressed image is 12. A method which gives a low value of SNR would mean it has a high level of noise which is not desired in compression. A low value of bpppb and a high value of SNR signify a good compression technique.

Based on the Table 1, we can see that for all the AVIRIS images, the BEZW technique gives the lowest bpppb value. For Cuprite, Jasper, Lunar Lake and Low Altitude, the values of 3D-SPECK and 3D-SPIHT are nearly equal. The results for JPEG 2000 MC and AT-3D SPIHT vary a little among themselves but are almost the same. Even though the values of all the methods are nearly equal, the best result is achieved by BEZW technique. Considering the Jasper image in Table 2, we observe that for every bpppb the SNR of BEZW technique is higher than the other techniques.

Table 1. Lossless Results (in bpppb) for AVIRIS Images

\begin{tabular}{|c|c|c|c|c|}
\hline Coding Methods & Cuprite & Jasper & $\begin{array}{c}\text { Lunar } \\
\text { Lake }\end{array}$ & $\begin{array}{c}\text { Low } \\
\text { Altitude }\end{array}$ \\
\hline 3D-SPIHT [18] & 6.80 & 6.72 & 6.05 & 6.61 \\
\hline 3D-SPECK [7] & 6.73 & 6.70 & 5.96 & 6.38 \\
\hline 3D-BEZW [2] & 4.76 & 4.82 & 4.88 & 4.90 \\
\hline JPEG-1700MC [2] & 5.76 & 5.56 & 5.70 & 6.54 \\
\hline AT-3D SPIHT [18] & 5.30 & 5.97 & 5.25 & 6.80 \\
\hline
\end{tabular}

Table 2. SNR (DB) for Corresponding bpppb for JASPER Image

\begin{tabular}{|c|c|c|c|c|c|c|c|}
\hline \multirow{2}{*}{ Coding Methods } & \multicolumn{5}{|c|}{ BITS PER PIXEL PER BAND (bpppb) for JASPER image } \\
\cline { 2 - 8 } & 0.1 & 0.5 & 1.0 & 1.5 & 2.0 & 2.5 & 3.0 \\
\hline 3D-SPIHT [18] & 29.07 & 39.42 & 45.14 & 48.91 & 51.6 & 53.83 & 56.03 \\
\hline 3D-SPECK [7] & 30.11 & 40.13 & 45.50 & 49.25 & 52.18 & 54.27 & 56.39 \\
\hline 3D-BEZW [2] & 34.15 & 46.59 & 49.21 & 52.60 & 53.51 & 56.01 & 57.96 \\
\hline JPEG-1700MC [2] & 30.62 & 40.89 & 46.14 & 49.78 & 52.81 & 54.65 & 56.58 \\
\hline AT-3D SPIHT [18] & 30.34 & 40.67 & 46.07 & 49.50 & 52.53 & 54.51 & 56.65 \\
\hline
\end{tabular}

Also, the BEZW technique's compression rate is comparable with the best known techniques and it comprises the advantages of EZW and SPIHT technique which are the high efficiency of significant searching based on well-design tree structures and the simplification of the coding algorithm.

\section{Conclusion}

In this paper, several known algorithms have been discussed for an effective lossless compression of hyper spectral images namely-dual tree BEZW, 3D-SPECK and AT-3D SPIHT. The BEZW technique presents desirable results with bpppb as low as 4.76 and SNR as high as 57.96. Also, the computational cost is comparable with the other coding techniques. The 3D SPECK method can reduce memory in the coding process and can increase the coding speed effectively but it has a complex coder. The last method discussed was AT 3D-SPIHT which is a very useful technique for hyperspectral image compression. This technique 
adopts a more effective 3D zerotree structure but does not provide better results than BEZW method.

Thus, by comparing all the above mentioned techniques, results show that, the dual tree BEZW compression technique outperforms the other techniques i.e. 3D SPECK and AT-3D SPIHT. Also, 3D BEZW compression method combines the merits of EZW and SPIHT algorithms, and is computationally simpler for lossless compression. This algorithm has a simple coder and the search is based on well-designed tree structures.

\section{Acknowledgment}

This work is supported (in part) by the Defense Threat Reduction Agency, Basic Research Award \# HDTRA1-12-1-0033, NASA EPSCoR program, and the National Science Foundation (NSF) award \#EPS-IIA1301726. Any opinions, findings, conclusions, or recommendations expressed in the material are those of the author(s) and do not necessarily reflect the views of NSF.

\section{References}

[1] Christophe, E., Mailhes C., \& Duhamel, P. (Dec. 2008). Hyperspectral image compression: Adapting SPIHT and EZW to anisotropic 3-D wavelet coding. IEEE Transactions on Image Processing, 17(2), 23342346.

[2] Cheng K. J., \& Dill, J. (Sep. 2014). Lossy to lossless dual-tree BEZW compression for hyperspectral images. IEEE Trans. on GeoScience and Remote Sensing, 52(9), 5765-5770.

[3] Guangbo, N., Binwen, F., \& Hui, L. (2009). Onboard compression of hyperspectral imagery based on hybrid prediction. Proceedings of the Asia-Pacific Conference on Information Processing (pp. 164-167).

[4] Abrardo, A., Barni, M., \& Magli, E. (2010). Low-complexity lossy compression of hyperspectral images via informed quantization. Proceedings of IEEE ICIP.

[5] Penna, B., Tillo, T., Magli, E., \& Olmo, G. (May 2007). Transform coding techniques for lossy hyperspectral data compression. IEEE Transactions on Geoscience and Remote Sensing, 45(5), 1408-1421.

[6] Rucker, J. T., Fowler, J. E., \& Younan, N. H. (2005). JPEG1700 coding strategies for hyperspectral data. Proceedings of IEEE International Geoscience and Remote Sensing Symposium.

[7] Ngadiran, R., Boussakta, S., Bouridane A., \& Syarif, B. (2010). Hyperspectral Image Compression with Modified 3D SPECK. Proceedings of IEEE CSNDSP 2010.

[8] Shapiro, J. M. (Dec. 1993). Embedded image coding using zerotrees of wavelet coefficients. IEEE Trans. Signal Processing, 41(12), 3445-3462.

[9] Said, A., \& Pearlman, W. A. (June 1996). A new, fast, and efficient image codec based on set partitioning in hierarchical trees. IEEE Trans. Circuits Syst. Video Technol, 6(3), 243-249.

[10] Christophe, E., \& Pearlman, W. A. (Feb. 2008). Three dimensional SPIHT coding of volume images with random access and resolution scalability. Journal on Image Video Proceedings.

[11] Islam, A., \& Pearlman, W. A. (1999). An embedded and efficient low complexity hierarchical image coder. Proceedings of SPIE Conference on Visual Communications and Image Processing: Vol 3653 (pp. 294305).

[12] Cheng, K., \& Dill, J. (Feb. 15, 2013). Hyperspectral images lossless compression using the 3D binary EZW algorithm. Proceedings of Image Process, Algorithms Syst. XI, SPIE Conf. (pp. 865515-1-865515-8).

[13] Galli, L., \& Salzo, S. (2004). Lossless hyperspectral compression using KLT. Proceedings of IEEE IGARSS (p. 316).

[14] Cheng, K., \& Dill, J. (Apr. 29, 2013). Lossless to lossy compression for hyperspectral imagery based on wavelet and 3D binary EZW. Proceedings of Defense, Security, Sens., SPIE Conference, Baltimore, MD, USA.

[15] Cheng, K., \& Dill, J. (March, 2013). Efficient lossless compression for hyperspectral data based on integer 
wavelets and 3D binary EZW algorithm. Proceedings of ASPRS Conference.

[16] Khelifi, F., Kurugollu, F., \& Bouridane, A. (2008). SPECK based lossless multispectral image coding signal processing. IEEE Signal Processing Letters, 15.

[17] Airborne visible / infrared imaging spectrometer. Retrieved form the AVIRIS website: http://aviris.jpl.nasa.gov/html/aviris.overview.html'1708.

[18] Hou, Y., \& Liu, G. (2008). Lossy-to-Lossless compression of Hyperspectral Image using the Improved AT3D SPIHT Algorithm. IEEE Proceedings of ICCSSE.

[19] Xiong, Z. X., Wu, X. L., Cheng, S., \& Hua, J. P. (March 2003). Lossy to lossless compression of medical volumetric data using three- dimensional integer wavelet transforms. IEEE Trans. on Medical Imaging, 18(3), pp. 459-470.

[20] Cho, Y., \& Pearlman, W. A. (June 2007). Quantifying the coding performance of Zerotrees of wavelet coefficients: Degree k Zerotree. IEEE Trans. Signal Processing, 55(6).

[21] Christophe, E., \& Pearlman, W. A. (2008). Three dimensional SPIHT coding of hyperspectral images with random access and resolution scalability. Proceedings of Conference on Signal Systems and Computers.

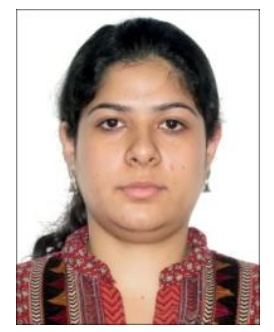

Astha Puri received her BS degree in electrical engineering from Chitkara University, Punjab, India in 2011. Currently, she is pursuing her master degree in the Department of Electrical and Computer Engineering, University of Nevada, Las Vegas (UNLV), Las Vegas, Nevada. Her research work is focused on climate data compression and hyperspectral images.

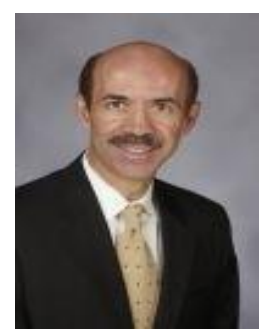

Shahram Latifi joined University of Nevada, Las Vegas, USA in 1989 after receiving his Ph.D. degree in electrical and computer engineering from Louisiana State University. He is currently a professor of electrical engineering at UNLV and has designed and taught graduate courses on bio-surveillance, image processing, computer networks, fault tolerant computing, and data compression in the past twenty years. He is a fellow of the IEEE society, he has been the recipient of several research awards including Barrick Scholar Award. He has published papers in high impact journals and is cited extensively by the global scientific community. He has been a PI- or co-PI on 25 grants totaling over 37 million dollars. His researches in several areas of information technology have contributed to southern Nevada's industries. 